\title{
Relationship between Size and Function of Natural Substance Particles
}

\author{
Yingge Zhang
}

Institute of Pharmacology and Toxicology and Key Laboratory of Nanopharmacology and Nanotoxicology, Academy of Beijing Medical Sciences. Beijing 100850, China

\begin{abstract}
All of the natural substances can be viewed as particles. According to the sizes of particles, the natural substances may be classified at six levels from small to large, and heir functional spectra can be established in the order from simple to complex. They are respectively point particles (?ppspPs), fundamental structural particles (FSPs), chemical particles (ChPs), nanoparticles (NPs), macroparticles (MPs) and celestial particles (CePs). The particles in one of these levels have their independent functional activities and interaction patterns different from those in the levels lower than them, representing a new leaping. There are very close relations between the size and functions of the particles. It is especially suitablefor biological particles. As far as the present human knowledges, the activity of the PPs is the simplest without interactions; the activities of FSPs are rather complex, their interactions result in the production of various FSPs; ChPs have more complex activities, their interactions produce inumberous sorts of ions and molecules, forming the collorful chemical world; the functional activities of NPs are much more complex than those of the ChPs, their interactions cause the production of inumberous sorts of biological NPs with the characteristics of biological phenomenon; the functional activities of MPs are the most complicated, their interactions result in the production of hundreds and southands sorts of individual living bodies with the feature of high level functional activities such as learnig, thinking and reproduction; we still know little about the functional activities of the CePs. In conclusion,the establishent of the size-function spectrum will be helpful to investigate the substance world as a whole, and reveal the interaction principles of the particles and biological essential fundamentals.
\end{abstract}

Keywords: Size-function relations, Fundamental particles, Chemical particles, Nanoparticles, Macroparticles, Celestial particles

Citation: Y. Zhang.Classification of substances based on use of nanoparticle as basical component. Nano Biomed. Eng. 2011, 3(1), 1-16. DOI: $10.5101 /$ nbe.v3i1.p1-16.

The establishment of the relations between substance structures and properties to reveal the principles governing their function changed with structures is one of the important methods to investigate the natural substances in the world. For example, the element periodical table greatly promoted the development of the chemical science. All the natural substances can be viewed as particles. Particle is one of the most fundamental modes of substance existence, although all the substance own both of the wave and particle properties. Size is also the most important feature of the substance particles. The natural substance particles can be divided at different levelsaccording to their sizes. The particles in different size levels have their own special movement and interaction principles. The illustration of the relations between the particle sizes and the principles of their movement and interactions is the results of the progress in human's knowledge about the natural substances. The analysis of these results will greatly deeply enhance our understanding of nanoscience and nanotechnology and life phenomenon, just like human's understanding of chemical elements and biological evolution through the establishment of elemental periodical table and Darvinism theory. Previously, the natural substance sizes were divided into three levels, that is, the micro-, midand cosmic levels, which failed to reveal the relations between the particle sizes and their functions, especially biological functions. In addition, there are various names for substance particles in different levels, such as fundamental particles and high energy particles for those particles in high energy physics, the atoms, molecules and ions for those particles in chemistry, the nanoparticles for those particles in nanoscience and nanotechnology, the animal in medicine and plant in botany, machines in manufacture and macroparticles in solid state science, and celestial bodies in astronology. These particle names are only the names called as a subject. There are confusions and disorders between the concepts of these particles, failing to give relevation for us to understand the substance world as a whole. This issue has been addressed in monographNanotoxicology" [1]. In this paper, we propose a new view or concept that to view all substances 
http://nanobe.org

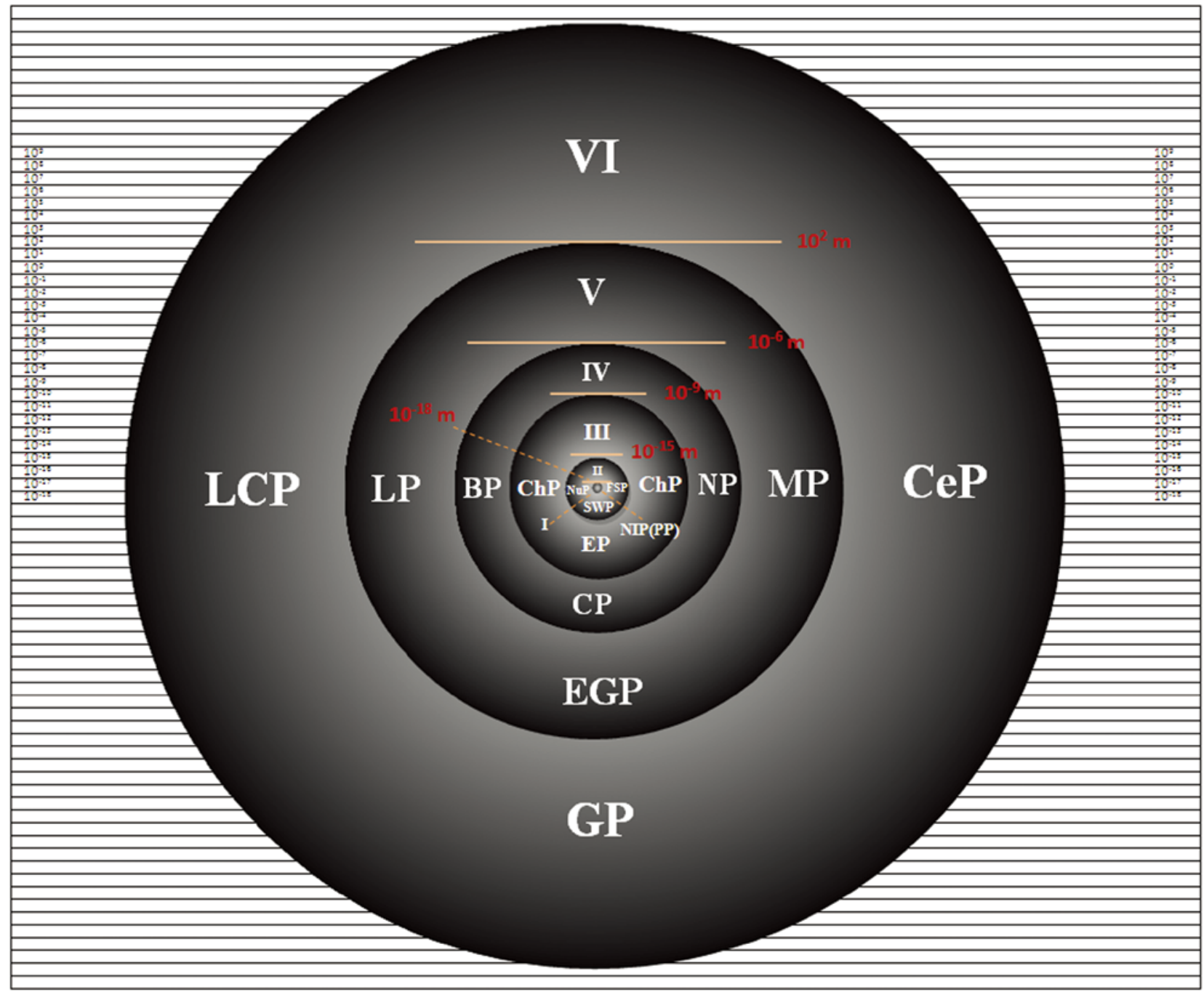

Fig. 1 The ball for Schematic expression of the size levels of substance particles The ball symbolize the particle model that is spherical without limits and composed of six size-levels of the particles from center to surface. The scale levels of substance particles are symbolized in the up direction. The sizes of the particles in level I $\sim$ VI are respectively $<10^{-18}, 10^{-18} \sim 10^{-15} \mathrm{~m}, 10^{-15} \sim 10^{-9}, 10^{-9} \sim 10^{-6} \mathrm{~m}, 10^{-6} \sim 10^{2} \mathrm{~m}$ and $>10^{2} \mathrm{~m}$. The custom names of the particles in different levels are symbolized in the right direction. The point particles (PPs) are smallest at the center (Level I) and the celestial particles (CePs) are largest in the surface of the ball (Level VI) while the fundamental structure particles (FP), the chemical particles (ChPs), nanoparticles (NPs) and macroscopic particles (MPs) are in turn larger and larger in the second (Level II), third (Level III), fourth (Level IV) and fifth (Level V) layers of the ball. The biology-based names are symbolized in the left direction from the center to the surface, in which they are in turn PPs at the center, nucleic particles (NuPs) Level II, ChPs in level III, biological particles (BPs) in level IV, life particles (LPs) level V and life carrying particles (LCPs) in level VI. The interaction-mode-based names are symbolized in the down direction from the center to the bottom, in which they are in turn no-interaction particles (NIPs) in level I, strong and weak interation particles (SWPs) in level II, electromagnetic interaction particles (EPs) in level III, complex interaction particles (CPs) in level IV, electrogravity interaction particles (EGPs) in level V and gravity interaction particles (GPs) in level VI.

as particles, to divide all of the natural substance particles into six levels, and investigate the relations between the particle sizes and their functions, especially biological functions, and try to establish a particle size-function spectrum based on present knowledge on natural substance particles.

\section{The division of particle size levels.}

All substances exist in particles [2], despite their shapes and morphology. In the viewpoint of particles, the natural substance can be divided into six size levels, which can be schematically expressed in a big ball (Fig. 1. Please pay attention to the unlimitedness of the ball although there is a board around it in the figure). The first level is the point particles (PPs), of which the diameters are $<10^{-18} \mathrm{~m}$ (Fig. 1I); the second level is Fundamental structural particles (FPs) including protons and neutrons, of which the feature size is between $10^{-18} \sim 10^{-15} \mathrm{~m}$ (Fig. 1II); the third level is chemical particles (ChPs), of which the feature size is $10^{-15} \sim 10^{-9} \mathrm{~m}$ (Fig. 1III); The fourth level is nanoparticles (NPs), of which the feature size is $10^{-9} \sim 10^{-6} \mathrm{~m}$ (Fig. 1IV); the fifth level is macroparticles (MPs), of which the feature size is $10^{-6} \sim 10^{2} \mathrm{~m}$ (Fig. 1V); the sixth level is celestial particles (CePs), of which the feature size is $>10^{3} \mathrm{~m}$ (Fig. 1VI). As far as the present knoledge the point particles are the tiniest particles, the fundamental structural particles can absorb or emit point particles, the chemical particles are made up of 
fundamental structural particles, the nanoparticles are of chemical particles, the macroparticles are of nanoparticles and the celestial particles are of macroparticles. The particles in each level have their own special movement and interaction principles. There are little or no intercrosss? between these principles in different levels. The complexity of their movement and interactions tends to increase as the size increases.

\subsection{Point particles}

We view point particles (PPs) as the first level particles that are the smallest ones, whichre believed to be undividable particles without recognizable spacial volume, observable inner structures and detectable mass. They have no interactions with each other and move freely in light speed and are not module particles of any other particles. Presently, the known PPs are photons. Electrons are not classified into PPs because they have detectable mass and are the module particles of atoms and their movement is generally bound to around the atomic nucleus although they have a diameter smaller than $0.002 \mathrm{fm}$ even accelerated to $100 \mathrm{GeV}$ [3]. PPs act mainly as the media particles in the reactions between particles. The known photons are the media particles of electromaganetic interactions[2]. The media particles for gravity may be gravitons, but this has still not been found yet[2].

\subsection{Fundamental structural particles}

Fundamental structural particles (FPs) are the structural basis of all substances, taking part in particle reactions, and including all of the particles that was called fundamental particles in the early 20 century, such as electrons, protons, neutrons, mesotrons, gluons, and so on. These particles are also called nucleic particles because the atomic nucleus are composed of them. The tiniest FPs are electrons with a diameter of no larger than 0.002 $\mathrm{fm}$, in the order of $10^{-18} \mathrm{~m}$. The upper limit of the sizes of FPs decreased by $10^{5}$ folds in comparison with that of chemical particles to the level of $10^{-15} \mathrm{~m}$. The largest FP is proton that is also the smallest chemical particles. With the development of techniques, studies on these particles have revealed that the microstructures of these "fundamental particles" do not belong to the same level in sizes and may be composed of deeper level particles. For example, the protons and neutrons are composed of quarks. Therefore, the word "fundamental" has been removed and all of these particles have been called particles, and the science about them has been insteaded by called particle physics since sixties of the 20 century. Currently we use "fundamental structural particles (FPs)" as the name of the particles that are studied in the field of particle physics to avoid the confusion with general particles that are studied in other scientific fields. Here FSPs also indicate the particles with diameters of $10^{-18}$ $\sim 10^{-15} \mathrm{~m}$ that are the presently-known tiniest structural basis of all of the substance particles.

\subsection{Chemical particles}

The third level was chemical particles (ChPs), that is, theose particles that have chemical particles, and take part in chemical reactions. ChPs including atoms, ions and molecules is a new name to distinguish these particles from theose particles in other levels. Atoms are the smallest particle units that keep the chemical properties of the substance. There are 114 chemical elements with several hundreds of atoms. The feature size of ChPs is $10^{-15} \sim 10^{-10} \mathrm{~m}$. Hydrogen atoms are smallest atoms and become protons with a diameter of $10^{-15} \mathrm{~m}$ when their only out-of-nuclear electron is lost so that the lower limit of ChP scale is $10^{-15} \mathrm{~m}$. The largest ChPs are those molecules made up of atoms. Myriad inorganic substance molecules including organic and inorganic ones have sizes in the order of $10^{-10} \mathrm{~m}$ smaller than $10^{-9} \mathrm{~m}$. Therefore, the upper limit of ChPs are defined as $10^{-9} \mathrm{~m}$. Some large organic molecules, such as macromolecular compounds, proteins and nucleic acids, are manufactured by polymerization or complex biological reactions and have nanometer scales. The movement and the interactions they experienced are obviously different from that of the small molecules. And therefore these large molecules were classified into nanoparticles. It is FPs that make up ChPs but the ChPs are so stable that they do not change their inner structures in chemical reactions, with exception of the loss of the outmost electrons or accept of the electrons by the surface electron layer of the atoms. In chemical reactions, the only event is the exchange of the outmost electrons between chemical particles while the inner FPs that composed the very few ChPs do not know what happens in the atoms, where they exist. Atoms are the ChPs that human have studied and known most clearly. Nowadays human know about several hundreds of atoms composed of 114 chemical elements, which can be listed in periodic tables according to their structures and the relations between their structures and properties. From the position of the atoms in the periodic table, human can know their atomic structures, including the number of the FSPs (neutrons and protons) in the nucleus, their atomic mass, the number of electron layers, how many electrons in each layer and the basic properties in chemical reactions. Molecules are those larger ChPs composed of atoms. Although there are only more than 100 chemical elements, molecules are innumerous because they are composed of atoms with different numbers and modes, making the number of molecules so large that we still can't know how many molecules in the natural world even with modern technology.

\subsection{Nanoparticles}

The third level particles are nanoparticles (NPs). These particles take part in the biological reactions in life activities. It is in this size level that life phenomenon 
develop so that NPs may be pertinently called biological particles. The sizes of NPs are similar with that of biomacromolecules such as proteins and nucleic acids. From the viewpoint of particles, NPs are composed of ChPs, just like ChPs are of FSPs. It is always true that the particles in the lower levels make up the particles in the higher levels. According to the definition of nanoscience and technology, the feature scale of NPs are $10^{-9} \sim 10^{-6} \mathrm{~m}$, namely $1 \sim 100 \mathrm{~nm}$. The sizes of nearly all biomacromolecules fall within this scale range. NPs have very active functional activities. It is in this size level that the life phenomenon take place, representing a new leaping in the sense of biology. Those NPs with biological activities and functions are called biomacromolecules, which are composed of ChPs but have properties different from ChPs. Proteins and nucleic acids are such biomacromolecules that exist in nature. It is right to believe that the fourth level is indeed the biomacromolecular level. In addition to the natural NPs, there are, with the rapid development of nanoscience and nanotechnology, more and more man-made nanoparticles with various complex and strong functions, such as carbon nanotubes [4], fullerenes [5], smart drug carriers [6] and so on. Also, the fourth particle level, namely the nanoparticle level is the level where human's micro/ nano-manufacture techniques will play sufficient roles. The nano world will be as colorful as the chemical world in not too long a time. Nowadays some scientists have proposed to establish the periodic table of some biomacromolecules [7], which means that it is possible for people to master and utilize the structure-function relations of NPs as well as that of ChPs. Generally, nanoscale is believed to be $0.1 \sim 100 \mathrm{~nm}$ [8-11], which is based on weather some special physical or chemical properties such as quantum effects develop. In the fields of biomedicine, however, what is more concerned on is the biological effects of the particles rather than simple physical and chemical properties. Many advantageous biological properties may be obtained under larger scales such as $280 \mathrm{~nm}$ [12] so that the definition of nanoscale is $1 \sim 1000 \mathrm{~nm}$ in biomedicine, which is also rationale. So, the upper limit of the sizes of NPs is here defined as $10^{-6} \mathrm{~m}$.

\subsection{Macroparticles}

The fifth level particles are macroparticles (MPs). MPs are higher level particles, which may be composed of NPs through very complex modes. The living bodies are one of MPs with the highest level functional activities. For the identification of activities and function with particle levels, MPs are defined as the particles that human can see with naked eyes or can see in the aid of simple magnifying tools such as magnifier or light microscope. The feature size of the MPs is $10^{-6} \sim 10^{2} \mathrm{~m}$. The lower limit of the definition is based on the scale on which the individual life bodies develop. For example, some singlecell organism bacteria and protozoon have the sizes about $1 \mu \mathrm{m}$ or $1000 \mathrm{~nm}$, equal to the upper limit of NPs.
The upper limit of the definition of MPs is not precise because the largest object that human can see depends on the distance from it. For example, the star we see may be very large although we feel small. Therefore, here we define the upper limit of MPs based on the scale of the subject that we can see its contour of the whole body as well as recognize its details, such as a stone that we can see its whole body as well as its thin particles on the surface. In addition, the smallest celestial body is seen on the scale of $10^{2} \mathrm{~m}$ [13], which may also be considered as the basis for the definition of the upper limit of MPs. From the viewpoint of particles, the macroscopic bodies are merely large particles, namely the so-called MPs. MPs are composed of NPs but have much lively activities and stronger functions, to which that of NPs are incomparable. MPs are very familiar to human and can be called life particles because of the developments of the living bodies in this size level.

\subsection{Celestial particles}

The sixth level particles are celestial particles (CePs), which are composed of MPs. From the viewpoint of particles, the celestial bodies are also merely particles. What is different from other particles is only that they have much larger sizes. The lower limit of CePs is defined as $10^{2} \mathrm{~m}$ because the known smallest celestial body has a diameter of $975 \mathrm{~m}$ [13], namely about $10^{3} \mathrm{~m}$. The upper limit cannot be defined presently. The sun is the largest particles in solar system where we live. The radius of the sun is $696100 \mathrm{~km}$, about 109 times of that of the earth on which we exist. The solar system is composed of the sun in the center and the nine planets revolving around it. Outside the solar system is the milk way system and outside the milk way system is many other star systems. What is there in the place beyond our vision? It may be conceived that there are merely various celestial particles in the unlimited time-space. As for the activities and functions, we still know little about them.

\section{The interactions between substance particles within individual levels}

The interactions between the particles within individual levels have general characters as well as individual features. The general character manifests as the dependence of the interactions on the media particles and the individual features lies in their special media particles for the interactions between the particles within a level. Here we discuss the individual characteristics within a size level.

\subsection{The interactions between PPs in the first level}

The known PPs are photons according to the defination abovedmentioned. There are no interactions between 
themselves so they are also may be called non-interaction particles (NIP in Fig. 1), but photons can interact with other particles. Photons are the bearer of energy and can interact with other particles in the way of energy transmission. For example, photons can challenge electrons, making the challenged electrons jump from a lower energy orbit to higher ones and electrons can emit photons when they fall from a higher energy orbit to a lower one.

\subsection{The interactions between FPs in the second level}

The interactions between FPs in the second level are governed by strong interactions and weak interactions so that they are called S-W particles (SWPs, Fig. 1). The strong and weak interactions depend on mesotrons and gluons [2]. It is through the exchange of media particles, FPs realize particle reactions such as fusion, fission, decay, annihilation, capturation, and so on, forming 479 FPs and the atomic nucleus of 114 chemical elements. There are four basic modes of interactions between the particles in the second size level, including strong, electromagnetic, weak and gravity interactions. Different interactions have different media particles and different capability to capture and release media particles. In order to understand the reason why the particles in different size levels have different interactions, it is important to make clear that there are close relations between the interaction modes and the distance, that is, all of the interactions decrease with the distance between particles increase. The behavior that interactions decrease with distance can be described in term of force range of which the physical meaning is the effective distance of interactions. The value of force range $(\mathrm{L})$ is proportional to the reverse of media particle mass: $L=h / 2 \Pi m c$ (here $h$ is Plank Constant, $\mathrm{m}$ is particle mass and $\mathrm{c}$ is light speed). If $\mathrm{GeV}$ is the mass unit and fm the range unit, the value of force range may be obtained by dividing 0.197 with the mass of media particles: $\mathrm{L}=0.197 / \mathrm{m}$. The capability of particles to adsorb or release media particles is often described by interaction coupling constant. Different interactions have coupling constant much different from that of other interactions[3]. The media particles of strong interactions are mesotron and guon. The slightest mesotron of strong interactions is $\Pi$ mesotron, of which the mass is about 0.149 time that of protons, which is relatively very large. The force range of the strong interactions is a short range force with a value of $1.4 \mathrm{fm}$ $=1.4 \times 10^{-15} \mathrm{~m}$. The mesotron of the weak interactions is $\mathrm{W}$ gluon with positive or negative charge or $\mathrm{Z}$ gluon without charge. Both $\mathrm{W}$ and $\mathrm{Z}$ particles bear large static mass. W particles have a static mass of about 85.5 times of protons and $\mathrm{Z}$ particles have that of about 97.2 times of protons. It is the huge mass that determinates the very short force range of the weak interaction, which is about 2.4 $X 10^{-18} \mathrm{~m}, 3$ orders shorter than that of strong interactions. The media particles in electromagnetic interactions are photons. Up to now, human have not found the media particles of gravity interactions. It should be graviton if it exists. Both photon and graviton have a zero static mass so that electromagnetic and gravity interactions are both long range interactions with an unlimited force range. These two interactions decrease relatively slower with distance and therefore can be studied under macroscopic conditions and have been clear in last century. It is because of the short range of strong and weak interactions and the small sizes of the particles in the second level that why these two interactions only play roles in the interactions between second level particles. Generally, there is only one media particle that takes part in one particle reaction between FPs[3].

\subsection{The interactions between ChPs in the third level}

In the third level, the strong and weak interaction both fail to function because their short effective range do not meet the distances between the particles. The interactions between the particles, namely the interactions between $\mathrm{ChPs}$, are mainly electromagnetic interactions (Fig. 2A) so they are called Eps based on interactions (Fig. 1). The media particles between ChPs are electrons. There are particle transformation between ChPs or changes in the relations of subordination at the same time as their structural changes in chemical reactions. The transformation and share of this particle between ChPs makes the electric force develop, so it is quite pertinent to say that this particle is the media particle of the interactions between ChPs. It is electrons that what is transported or shared between ChPs. For example, when a natrium atom meets with a chlorine atom, the electron in the outmost electron layer of the natrium atom is captured by the outmost electron layer of the chlorine atom, which makes the static electric interaction develops between the two atoms, leading to the formation of a natrium chloride molecule (Fig. 2A); during the chemical reaction of hydrogen oxygenation to form $\mathrm{H}_{2} \mathrm{O}$ molecule, the electrons of the two hydrogen atoms changes their subordination status from complete subordination to hydrogen atoms alone to sharing of them with one oxygen atom, which makes covalent and static electric force develop between hydrogen and oxygen atoms (Fig. $2 \mathrm{~A}$ ); during the reactions for the formation of some gas molecules, the valent electrons that originally belong to one atom alone become sharing between two atoms, such as the formation of oxygen and hydrogen molecules from their atoms (Fig. 2A). Nearly all of the chemical reactions and the keeping of molecule integrity, namely the interactions between ChPs, are mediated by the exchange, re-distribution or sharing of electrons between atoms. Some large particles, such as basic chemical or functional groups, are secondary ChPs of some basic reactions, which can take part in chemical reactions as undividable ChPs, being also because the sharing and re-distribution of the covalent electrons between the module atoms, 
http://nanobe.org

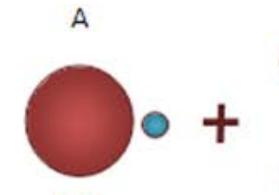

$\mathrm{Na}$
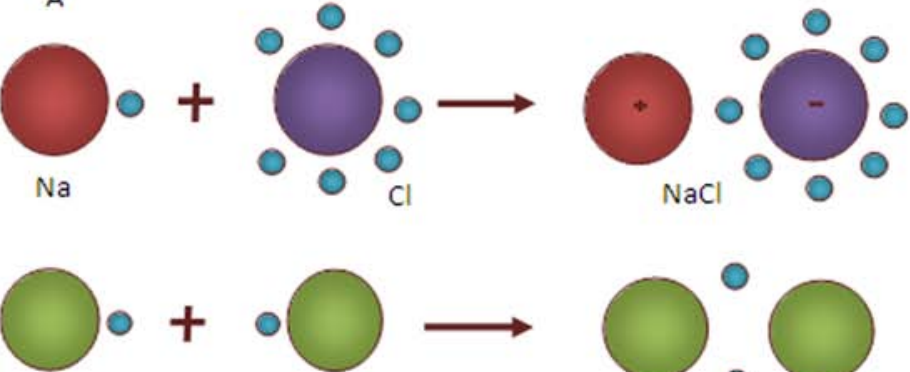

H

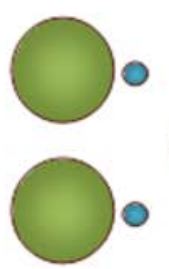

$\mathrm{H}$

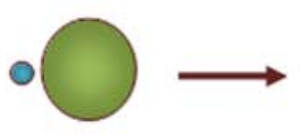

$\mathrm{H}$

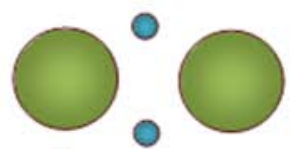

$\mathrm{H}_{2}$

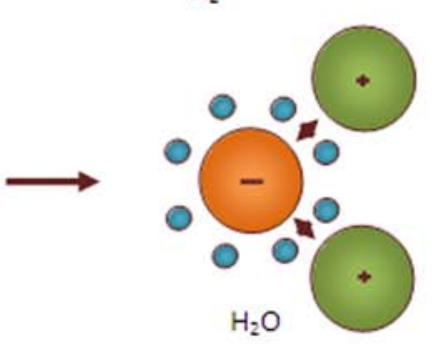

O
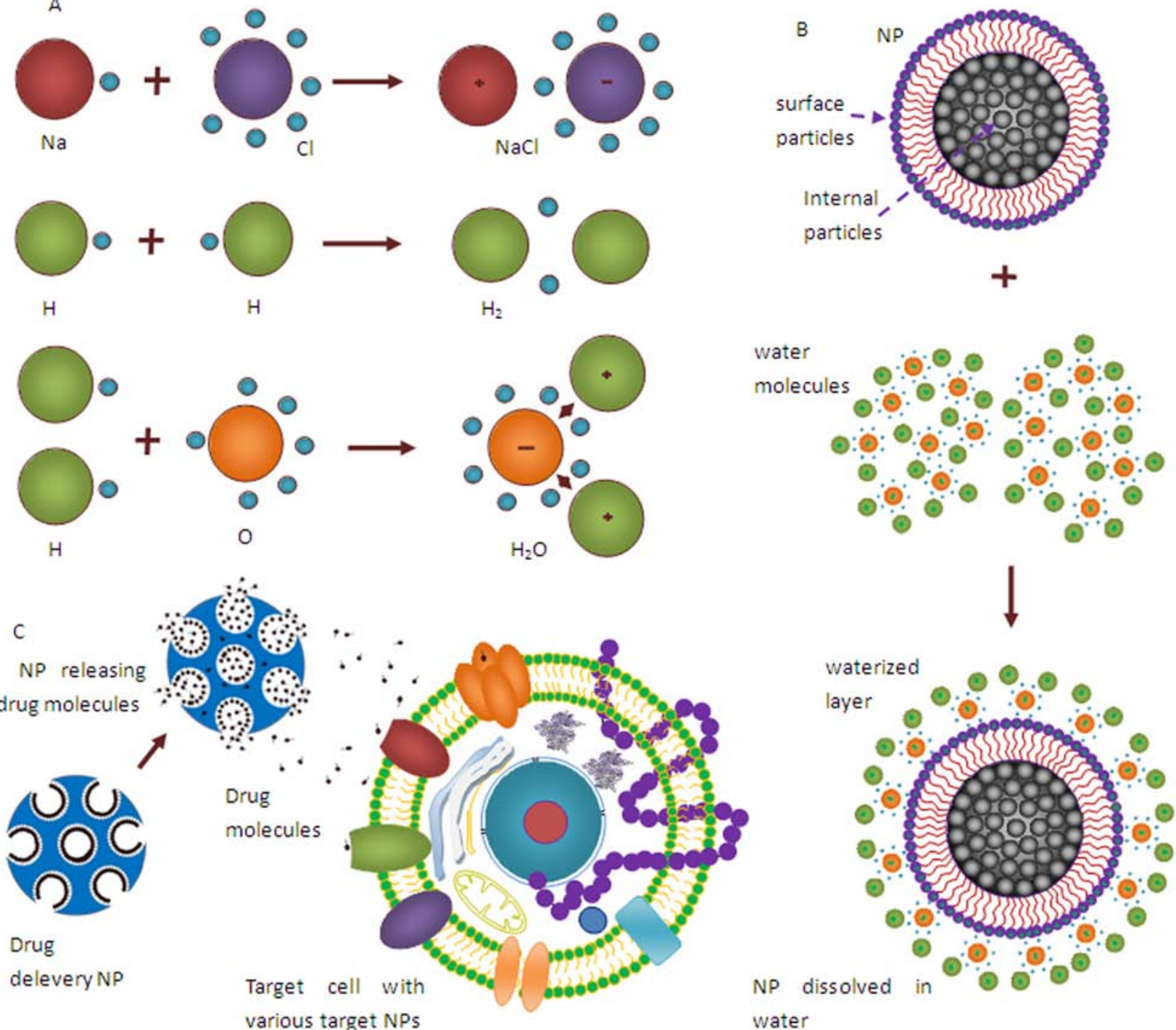

various target NPS

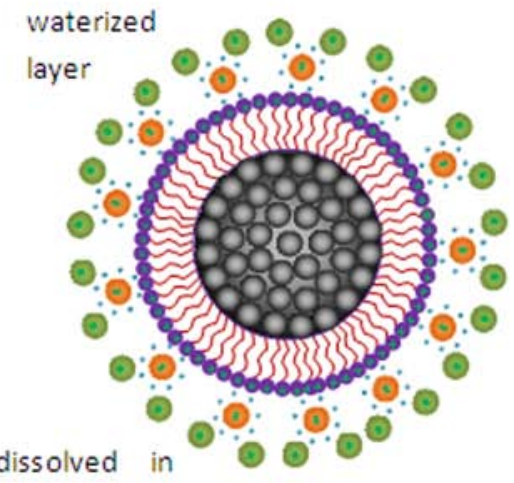

water

Fig. 2 The comparison of the dependence of NPs on media particles with that of ChPs A: ChPs take electrons as their media particles of interactions: upper shows the case of media aprticle transformation. The out most electron of the natrium atom is lost and obtained by a chlorium atom, making the natrium atom bear positive charge and the chlorium atom bear negative charge so that electronic force develops between the 2 atoms. The middle and the bottom show the case of media particle shared between atoms. The share of electrons between 2 hydrogen atoms produce combine force between the 2 hydrogen atoms to form a hydrogen molecule (middle). The uneven share of electrons between 2 hydrogen atoms and 1 oxygen atom produce not only the covalent combination force but also electronic force, leading to the formation of water molecule. B: NPs depend on the surface ChPs to interact with its environmental substance particles: a NP with surface hydrophilic molecules is dissoved in water by positively charged surface ChPs interacting with water molecules to form a waterized layer, with the medial ChPs in the surface nott leaving NPs. C: A NP with drug molecules asemplized on the surface in the micropores of mesoporous it. The drug molecules leave the NP for the target cell when the drug delivery NPs to develop curative effects.

making special large combination forces develop between them. From these facts, it may be seen that electrons are the media particles for the interactions between ChPs. The similarity of ChPs and FSPs in interactions may be considered in the context of the exchanges of media particles between the particles within a size level, that is, both of the in-level particles interact in dependence on the exchange of media particles. What is different is only the difference in the media particles or the properties of them. Considering the size, the media particles for the interactions of ChPs are much smaller than that of FPs. The mass of electrons is only $1 / 1840$ of protons, while the mass of most FSPs is between $200 \sim 21600$ times of that of electron mass and the mass of the media particles for the interactions of FPs are also much larger than the mass of electrons. For example, the mass of $\mathrm{W}$ particles is about 15732 times of electrons and that of $\mathrm{Z}$ particles is more than 16000 times of electrons; Even the mass of the lighest $Л$ particles of strong interactions is 274 times stronger than that of electrons. In the ChP level, there may be more than one media particles (generally $1 \sim 7$ ) to take part in one of the chemical reactions. Of course, the role of electrons in some chemical interactions still depends on photons but the ChPs interactions directly depend on electrons after all. 


\subsection{The interactions between NPs in the fourth level}

The interactions between the fourth level particles, namely NPs, are dependent of ChPs, of which NPs are composed. The forces governing the interaction between NPs are a composite of many intermolecular forces such as hydrophobic forces, electrostatic forces, van der Waal's forces and hydrogen bond, so they are called composite interaction particles (CPs, Fig. 1). It may be said that NPs take ChPs as their media particles in all of the interactions between NPs. The sizes of NPs increase obviously in comparison with that of ChPs. One NP may contain many ChPs, of which the number may get thousands or even more. These myriad module ChPs may connect with each other by the head of one and the tail of another to form straight chains first, then fold up into oligomers with special three-dimensional structures and further polymerize into polymers as proteins. Some ChPs may form complex branch structures with a large size such as glycogen. Man-made NPs may be composed of only one kind of module ChPs with simple structures or of several kinds of module ChPs with complex structures. Due to the large sizes, some of the module ChPs are in the surface while the others in the center of NPs. Only the module particles on the surface of NPs change their structures when nanoparticles interact with each other or with their environment [14]. The internal module particles do not directly take part in the interactions between NPs or between NPs and their environment. The roles of the surface module particles are very like the electrons in the outmost electron layer of ChPs, for which the best example is the interactions between enzymes and their substrates. A part of the surface groups of the enzyme $\mathrm{NPs}$, as the module ChPs, go together to form special local structural domain as the active center, namely, the site that directly takes part in functional activities [15]. The surface ChPs serve as the media particles of the interactions but they do not leave the particles (Fig. 2B), unlike the media particles for the interactions of FSPs and ChPs, which is a new phenomenon that appears in the fourth size level particles. The dependence of manmade NPs on their surface module ChPs is much more obviously, such as in the case of drug delivery systems. The present drug delivery systems often use NPs, such as mesoporous NPs, as the drug carriers [16]. In such a case, the whole system is indeed a NP with the drugs as the media particles to develop curative effects. The drug molecules leave NPs for their target cells or molecules to develop their curative effects (Fig. 2C). As mentioned above, there are two cases that the dependence of NPs on the media ChPs in their actions on their environment, one of which is the media ChPs do not leave the surface of NPs when interactions develop and the other of which is the media ChPs are released from NPs into the environment and their effects develop when these media particles diffuse to the target substances [17]. Of course, these media ChPs are dependent on electrons for their role in the interactions of NPs with each other or with their environment, but the dependence of NPs on the ChPs is direct and that on electrons is indirect after all. In some cases, NPs can use electrons as media particles, such as in the case of the NPs that their module ChPs combine with each other by metal? bond. In the fourth particle level, there are many media ChPs to take part in the interactions of NPs, not like in the case of second level particles. Many intermolecular interaction forces, such as hydrophobic forces, electrostatic forces and hydrogen bonds, develop between the module particles of NPs to keep the integration of module particles together as whole particles with stable functional steric structures.

\subsection{The interactions between MPs in the fifth level}

The interactions between MPs and between these particles and their environment in the fifth level are mainly electromagnetic and gravity interactions (therefore they are called EGPs based on interactions, Fig. 1), much more complex than that of the particles in the abovementioned four levels, but they still need the particles in the lower size levels as the media particles. It is in this level that the individual life bodies appear, which symbolizes the main features of the MPs in the fourth size level. These life bodies may be composed of only one cell or of innumerous cells. On the surface of each life body, there is a biological membrane (in single-cell organisms) or skin (in multicellular organisms) to enclose its internal structures from the outside environment to keep its contents stable and in a good order of metabolism. To realize the life activities including assimilation, dissimilation and proliferation, the interactions between these life bodies and between the life bodies and their environment are extremely complex and dependent on various ChPs and NPs. The cell membranes are double layer lipid membranes with a thickness of about $2 \mathrm{~nm}$, through which the interactions of single-cell organisms with environment take place. The membranes are composed of lipid ChPs and inlaid by many functional protein NPs such as receptors, ion channels, antigen and enzymes. All of these ChPs and protein NPs may be considered as the media particles of the interactions of the tiny life body with its environment. These media particles either leave the particles or stay in the membranes during the mediation of interactions, like the media ChPs on the surface of NPs. The skin are a special tissues composed of many highly differentiated cells, which provide protection of the internal contents of the life bodies and take part in the interactions of life bodies with environment. The larger particles in the fifth level have more complex interactions with their environment, for which various glands are best examples. The exocrine gland can secrete various biological NPs to realize their action on the outside substances. For example, the digestion gland can secrete digestive enzymes into the gut to digest foods. The toxic glands of some animals such 
http://nanobe.org

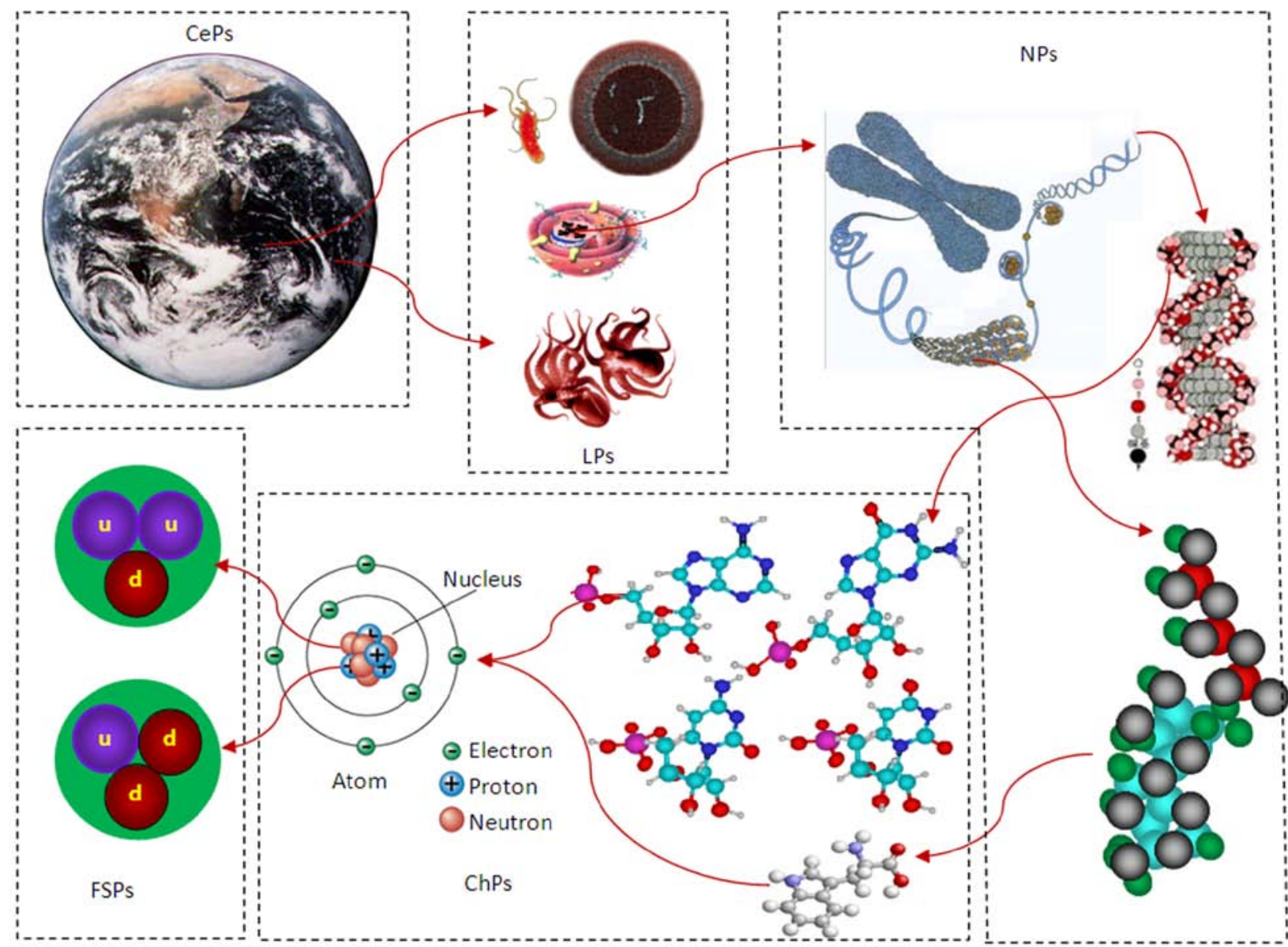

Fig. 3 The schematic structural relations between the substance particles CePs: celestial particles, which are partly composed of life particles (LPs). LPs are composed of biological nanoparticles (NPs). NPs are composed of chemical particles (ChPs). ChPs are composed of fundamental structural particles (FPs)

as snake can secrete toxins that can be injected into the other life bodies to cause the death of them. Because of the macroscopic sizes, the larger life bodies have various distinctive internal structures composed of various tissues and myriad cells. There are complex internal interactions between tissues and cells in life bodies. For example, the regulating actions of endocrine glands by secreting hormone that may be transported to remote sites in blood to develop their biological effects. Man-made inorganic MPs such as machines, airplanes and cars also have various complex interactions with each other and environment to realize their movements and functions. There are also more complex dependent relations between MPs. For example, human bodies are highly smart MPs that can manufacture and use inorganic MPs to investigate the nature, to change the nature and to tame the nature. So, the fifth level MPs may directly depend on other MPs, secondarily on NPs, thirdly on ChPs and fourthly on FSPs as media particles in interactions. The number of the media particles taking part in interactions may be much tremendous, much more than that in the fourth level. There are also electromagnetic and gravity interactions but they are not discussed here because these interactions have been very familiar to us and have been well utilized by human for various purposes.

\subsection{The interactions between CePs in the sixth level}

As far as we know at present, the interactions between celestial bodies are mainly gravity interactions [2]. The media particles of the gravity interactions are gravitons, which have been not found up to now. The cosmic structures are kept by gravity. For example, the planets are bound to the periphery of the stars like the sun by gravity. Some celestial bodies such as the sun can emit light, by which to influence the other celestial bodies such as the earth. From broader sense, there is a mode of interactions between the celestial bodies depending on MPs. For example, human have been sent to some celestial bodies such as moon and mars. It may be conceived that these activities of human will become a mode of interactions between celestial bodies in future.

\section{The inter-level relations of substance particles}

While there are special movement and interaction principles between the particles within individual size levels, there are some inter-relations between the particles 


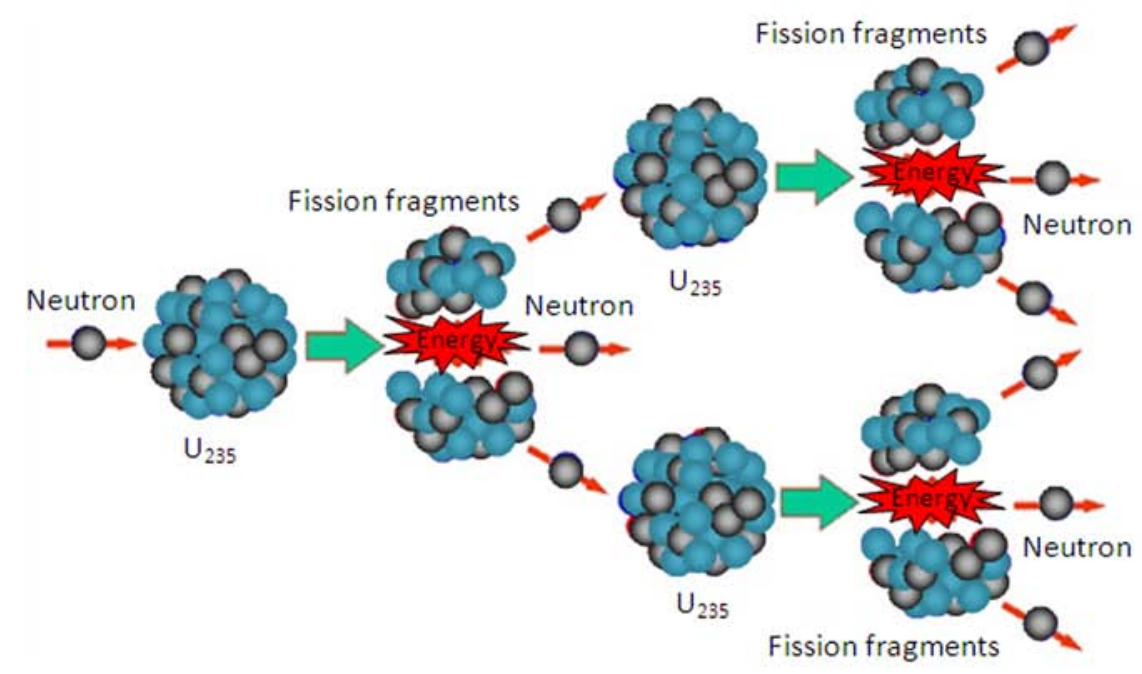

B

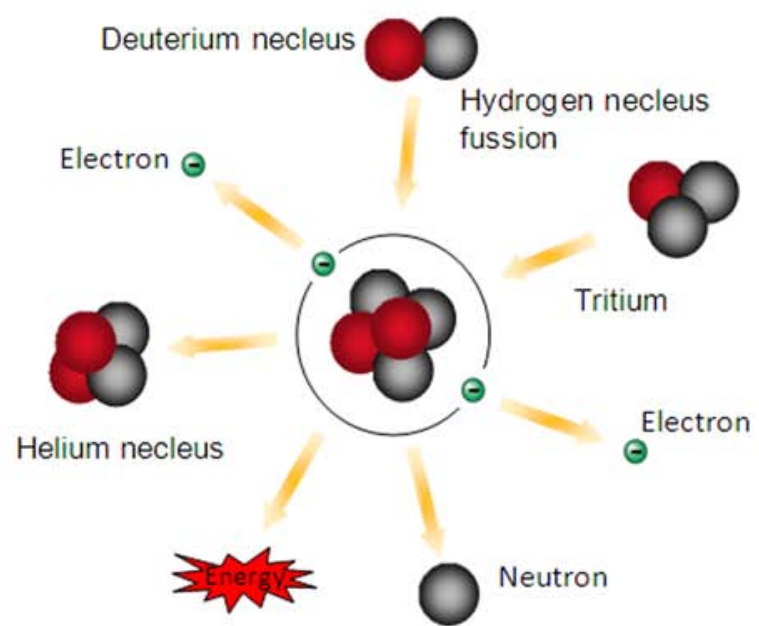

C

Fig. 4 The transformation of some particles in different size levels A: a heavy ChP (U235 nucleus) transform into other to ChPs and FPs (neutrons) through fission; B: two nucleuses of light ChPs transform into a helium nucleus (FP) and a neutron (FP); C: the example for the transformation between ChPs and NPs. In the process of protein synthesis, peptide bond forms in biochemical reactions. The two amino acids, between which a peptide bond forms, loss one hydrogen atom and one hydroxygen group respectively. It may be said that the original amino acids have disappeared and new NPs have developed when the synthesis of protein finished. Reversibly, in the process of protein disintegration, the disruption of the peptide bond are accompanied by the addition of one hydrogen atom and one hydroxygen group respectively to the two neighbouring amino acids. It may be said that the original NP has disappeared and two ChPs have developed

in different size levels. These inter-level relations include the structural relations, the transformation relations, the manufacture relations and the regulation relations

\subsection{The structural relations}

Structurally, the relations between the particles in different size levels are the relations of constitution, with the exception of PPs in the first size level. Generally, the higher level particles are consisted of lower level particles. There is an order for the inter-level structural relations of substance particles. CePs are consisted of MPs, MPs are of NPs, NPs are of ChPs, ChPs are of FSPs (Fig. 3). PPs are not structural particles but a kind of media particles. The inter-level structural relations are different from that of the in-level structural relations. In the in-level structures, the module particles are combined together by in-particle forces while those module particles are combined together by inter-particle forces in inter-level structures. For example, the protons and neutrons, 2 kinds of FPs, are combined together within an atomic nucleus, another kind of FPs, by in-nucleus (FSP) forces but the nucleic particles including photons and neutrons and the electrons are combined together by inter-FP forces in an atom, a $\mathrm{ChP}$; the atoms, one kind of ChPs, are combined together by in-molecule (ChP) forces in molecules, another kind of ChPs, but those ChPs in nanoparticles (NPs) are combined together by intermolecule forces. In proteins and nucleic acids the module 
http://nanobe.org
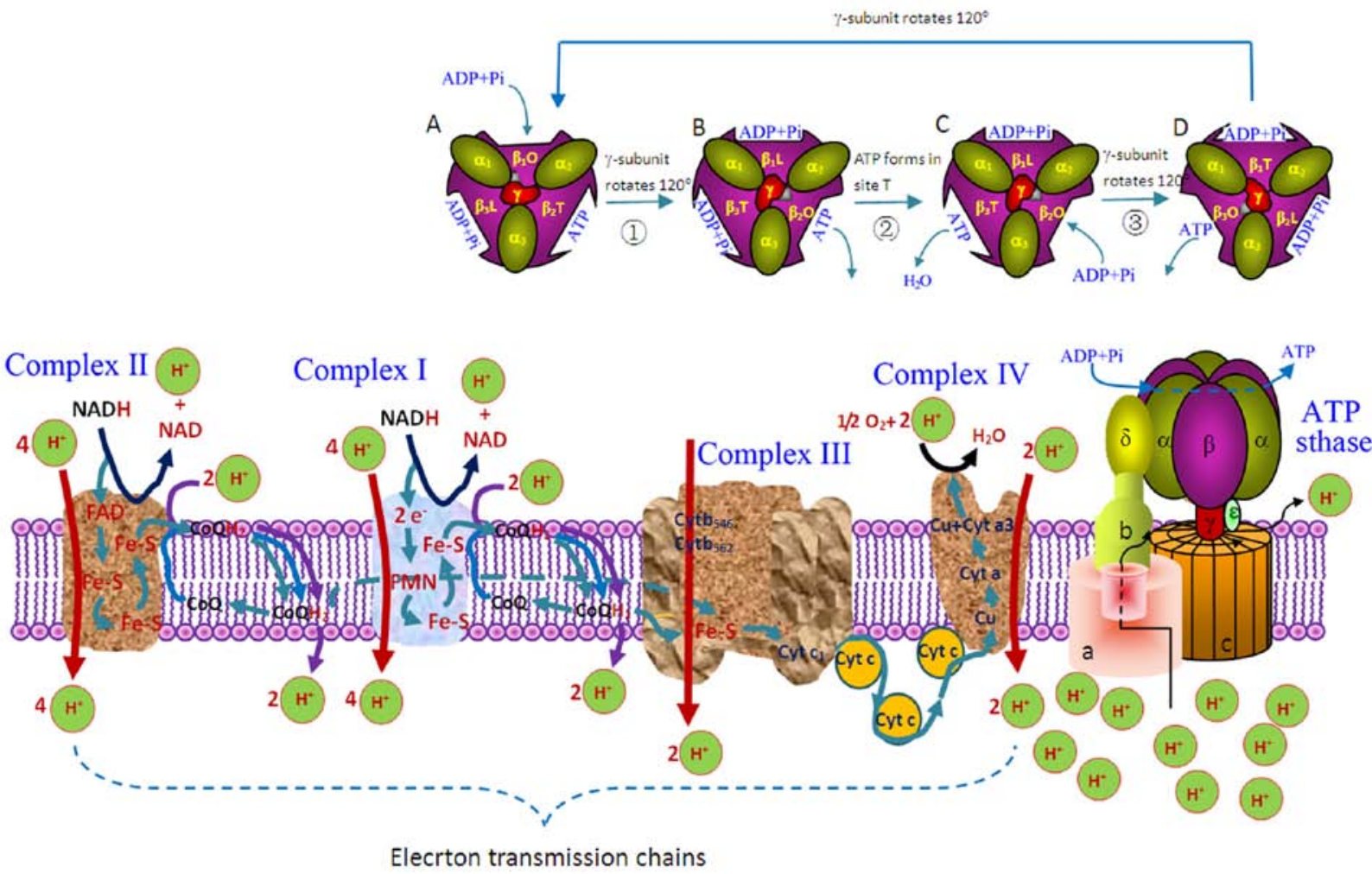

Fig. 5 The examples of NPs to manufacture ChPs with ChPs The ATP synthase, one of NPs composed of 23 subunits, manufactures ATP from ADP and inorganic phosphates (Pi). ATP, ADP and Pi are all ChPs. The rotation of the subnit $\gamma$ makes the $\beta$-units cycly and alternatively change their steric structures, which in turn take the conformations that respectively have the functions of high affinity for ADP and Pi, ATP synthasis and ATP release. As schematicly shown at above, when the $\gamma$-unit at a state as in A, $\beta 1$-subunit have an open conformation for ADP and Pi to bind and $\beta 2$ subunit takes the conformation of high affinity for ATP while $\beta 3$-subunit has low affinity for ADP and Pi. After the $\gamma$-unit rotates $120^{\circ}$ clockwisely (B), $\beta 1$-subunit conformstion changes from open state into the state of low affinity for ADP and Pi, $\beta 2$-subunit conformation changes from its high affinity for ATP into open state to release ATP from it, while $\beta 3$-subunit conformation changes from low affinity into high affinity for ADP and Pi. In $\beta 2$-subunit that has released ATP, another ADP and Pi come to bind and an ATP molecule was synthasized in high affinity $\beta 3$-subnunit (C). Finally, $\gamma$-unit again rotates $120^{\circ}$, now $\beta 1$-subunit become high affinity, $\beta 2$-subunit become low affinity and ATP is released from $\beta 3$-subunit (D), then $\gamma$-subunit continually rotates $120^{\circ}$, turning back to the state A. In this way ATP molecules are continually synthesized. The control and regulation of the ATP synthesis are realized through subunit $\gamma, \delta, \mathrm{a}, \mathrm{b}$ and $\mathrm{c}$. The energy to drive the rotation of $\gamma$-subunit is provided by the protons. The high concentration difference between the two sides of the mitochondrial membrane built up by the electron transmission chains drive the protons to pass through the channel in subunit $\mathrm{a}, \mathrm{b}$ and $\mathrm{c}$, which produce force to drive the rotation of the roter composed of subunit $\mathrm{c}, \gamma$ and $\varepsilon$. There are two electron transmission chains, of which one is composed of complex I, III and IV and the other is composed of complex II, III and IV.

ChPs combined together by covalent that may be called in-molecule forces if the proteins were considered as large molecules, but the covalent bond is still one kind of force between ChPs, an inter-ChPs force. In fact, the inparticle forces are the main factors to keep the particles integrated while the inter-particle forces are the main forces to make up higher level particles. The structural relations between NPs and MPs are much more complex than that between other 2 levels. Various biological NPs with different shape, properties and functions are combined together by inter-NPs forces to make up living MPs. Many forces play role in the building of living MPs. These forces in combination may be called bio-structural forces. For example, proteins, nucleic acids, lipid membranes, polysaccharides are combined together by complex biostructural forces. Although the biostrucutral forces require further studying, they may be composed of hydrophilic forces, hydrophobic forces[18], tissue pressures, surface tension forces, osmotic forces, electrostatic forces [18] and so on to form cells or singleor multiple-cell life bodies. The CePs are composed of MPs but we know little the detail structural relations between CePs and other level particles. PPs have no structural significance so they have no structural relations with other level particles.

\subsection{The transformation relations}

Transformation may develop in the particles in different size levels. Transformation is different from constitution. The transformation means the disappearance of the original particles and the production of new particles. Photons can be absorbed by other particles, resulting in the increase of the mass or energy of other particles, so the photons may be considered to have disappeared and transformed into a part of other particles although without the production of entire new particles. The transformation between FPs and ChPs can be understood easily because 
it has been a well known fact that larger nucleus (FPs) can transform into smaller nucleus through fission (Fig. 4A). The new nucleuses can become new atoms (ChPs). New atoms can from light nucleus through fusion (Fig. 4B). There seems to be some difficulties in understanding the transformation between ChPs and NPs. In most chemical or biochemical reactions, the ChPs may combine together to form NPs, which is what so called bottom-up method to prepare NPs. Some people may argue that the ChPs become module particles of NPs rather than disappear so that we only can say that the ChPs make up NPs rather than that ChPs transform into NPs but in the process of ChPs becoming NPs the original ChPs loss some of their atoms or groups so that the original ChPs may be considered to have disappeared while new NPs develop. For example, when amino acids are used to synthesize proteins, one of the neighbouring amino acids losses a hydrogen atom and the other losses a hydroxygen group, which means the two original amino acid particles have disappeared while a protein develops so that the protein synthesis may be considered as a process of ChPs transforming into NPs (Fig. 4C). the transformation of biological NPs into ChPs is quite easy to understood -The disruption of biological NPs leads to the production of new ChPs, in which there is the disappearance of NPs and the production of ChPs. As for the transformation between MPs and NPs, a usually used method to prepare NPs is the top-down method, in which the MPs are break down into NPs with the disintegration of the MPs and the production of new NPs. Just like the case of ChPs to transform into NPs, NPs transform into MPs as the module particles of MPs rather than the disappearance of NPs. Up to day, we still don't know whether there is transformation between CePs and other particles. Nowadays, the studying of the transformation of ChPs and MPs into NPs is a hot point because they may become useful methods to prepare NPs.

\subsection{The manufacture relations}

Some particles can manufacture other particles. Not only particles can make the particles in the same size level but also that in other size level. The FPs can manufacture ChPs through their activities. For example, when alpha particles pass through biological tissues, they result in the ionization of biological molecules, and in this way new ChP ions are manufactured from ChP molecules [19-20]. The ionization effects of alpha particles on the tissues of life bodies have important toxicological significance, which has been a scientific field for a rather long time [21] and has also been used to the treatment of cancers [22-30]. The other FPs, such as beta particles and gamma particles can also manufacture new ChPs from old ones by disintegrating ChP molecules. NPs can manufacture ChPs. The synthetase, one kind of biological NPs, can manufacture larger chemical molecules, namely ChPs, from some smaller $\mathrm{ChP}$ molecules or manufacture smaller ChPs from larger ones. For example, ATP synthase can synthesize ATP molecules with ADP and inorganic phosphate [31], manufacturing larger ChPs from smaller ones (Fig. 5), and acetylcholinesterases can hydrolize acetycholine into acetyl acid and choline [32,33], manufacturing smaller ChPs from larger ones. Recently, it has been found that some inorganic NPs can manufacture ChPs. For example, ferromagnetic nanoparticles have Intrinsic peroxidase-like activity and can manufacture water and oxygen molecules from hydrogen peroxide [34]. More excitingly, some specialist deliberately designed NPs can manufacture various ChPs [35-38]. MPs, especially the life MPs, have much more potent functions to manufacture other particles-not only those smaller than themselves but also those much larger than themselves. The products manufactured by MPs can be divided into three classes. The first class includes those manufacturing enzymes as mentioned above and the regulating molecules such as hormones that play important roles in life activities and those excrine enzymes that are excreted to gastrointestinal tract to digest food contents and facilitated the absorption of nutrients. The second class is the small ChPs with various unilities, such as drugs. The third class is the replications of themselves. All of the life bodies can manufacture their decendants through sexual or non sexual proliferation to continuity of the nationality. The fourth class includes various tools manufactured by mankind, the highest level living MPs, including those used in transportation, space-traveling, minerization, war, and so on. CePs have also been made by human, such as sattelites and spaceships. We still don't know whether CePs can manufacture anything but the lives on the earth is realily a magic created by CePs. It is the energy from the sun that brings birth to all of the organisms.

\subsection{The regulation relations}

Without regulation and control, all of the machine would not run normally and all of the lives would not survive. Very complex regulation mechanisms exist between the particles in different size levels. Generally, the functions and activities of various particles are regulated and controlled by the particles smaller than themselves. The best examples to illustrate the regulating and control relations are that between ChPs and NPs and that between NPs and MPs in life bodies (Fig. 6). The regulation effects of ChPs on NPs are common mechanisms for normal life activities. There are activators, inhibitors and reactivators for enzymes, and there are agonists, antagonists and hemiantagonists for various receptors. For example, cAMP is reactivator of protein kinase and has regulation functions on the activities of the kinase (Fig. 6A) and $\mathrm{ACh}$ are agonist of cholinergic receptor and has control effects on the ion channel in the receptor (Fig. 6B). These enzymes and receptors can be regulated not only by the ChPs synthesized in bodies but also by those synthesized out of the bodies and therefore they are important targets of the drugs to cure the corresponding diseases. Above mentioned regulations develop between ChPs and 
http://nanobe.org
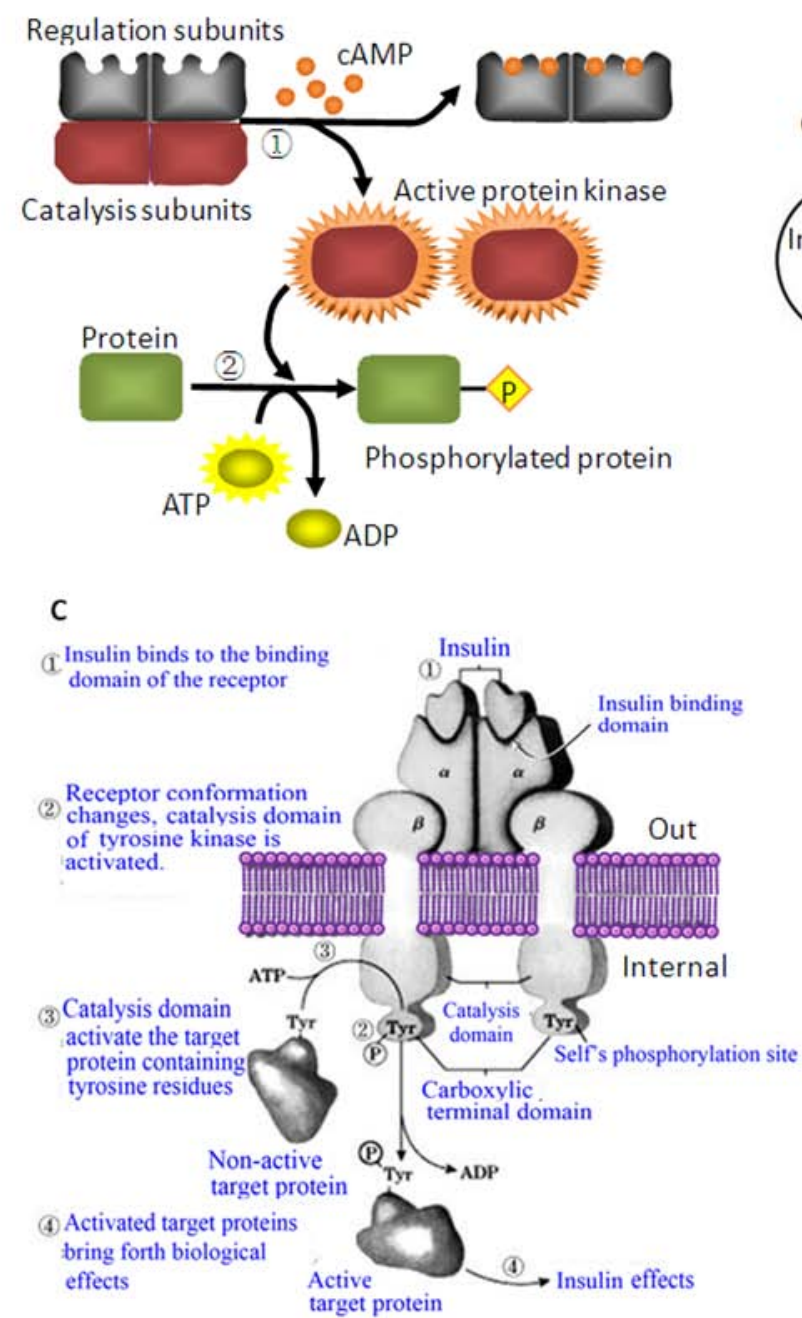

B

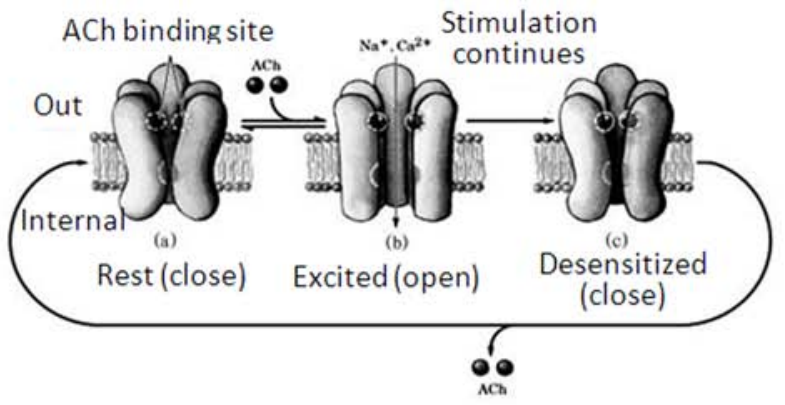

D

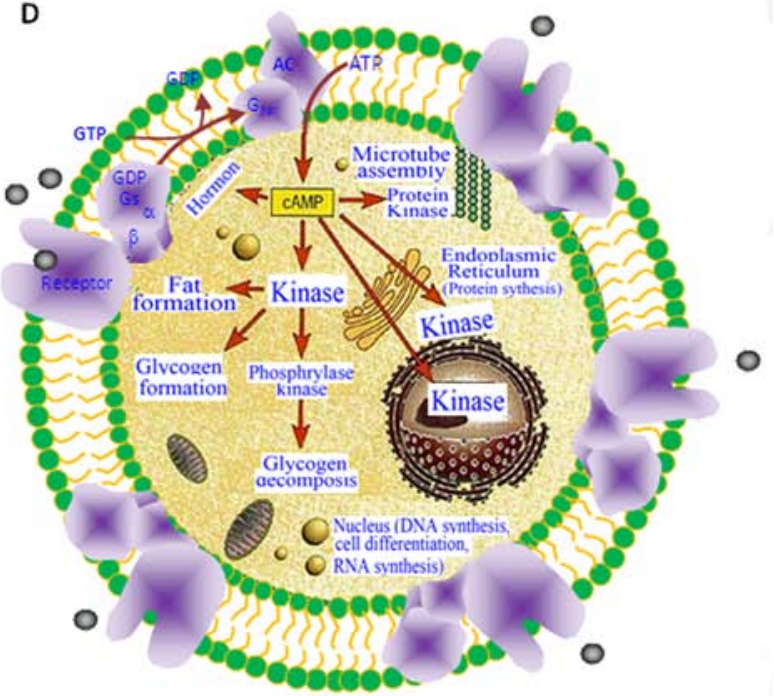

Fig. 6 The regulation and control relations between ChPs, NPs and MPs A: The relations of regulation relations between ChPs and NPs. Here the cAMP molecules, one kind of ChPs, are regulator of protein kinase, one kind of biological NPs. The Protein kinase are composed of two regulation subunits and two catalysis subunits. The binding of cAMP to the regulation subunits makes protein kinase change its conformation and the catalysis subunits datached from the regulation ones ((1)) and become active enzymes from the non-active ones. The active protein kinase can function to catalyze the phosphorylation of proteins ((2)). B: The control relations between ACh molecules, one kind of ChPs, and ACh receptors, one kind of NPs. The binding of ACh to the binding sites of the receptor, the central channel of the receptor opened, allowing the $\mathrm{Na}^{+}$and $\mathrm{Ca}^{2+}$ flow into cell through the channel to bring forth active potentials. This is a control relation between ChPs and NPs. C: The regulation relations between NPs and MPs. The binding of insulin, a kind of biological NPs, to the binding domain of insulin receptor, makes the receptor change its conformation and the catalysis domain of the receptor be activated. The activated catalysis domain can activate the target proteins containing tyrosine residues. The activated target proteins bring forth a series of biological effects. D: The system illustration of the regulation and control relations between ChPs, NPs and MPs (cells). The special living activities of the cell are controlled and triggered by the binding of ChPs such as epiephrin, which activate NPs. The various activated NPs bring forth a series of chemical and physical reactions and finally result in the ordered and entire living activity.

NPs. The regulating and control between NPs and MPs are much more complex such as the immunological regulating circles and the regulation effects of hormone on the activities of cells (Fig. 6C). The regulation and control of the functions of MPs are the most complex and need many particles in different levels, especially ChPs and NPs, through long way of signal transduction (Fig. 6D). For example, when an animal want to walk, the corresponding muscles must contract, which needs the neurotransmitters (ChPs) released from the nerve terminals, and the muscle contracts must be stopped by the elimination of the neurotransmitters, which is done by the enzymes called acetylcholinesterases, a biological NPs. Between the contact of neurotransmitters with the receptors and the contract of muscles, there is a complex signal transduction. PPs such as photons and FSPs such as alpha particles can influence the activities of enzymes but which are destroying effects rather than regulating effects.

\section{The functions of the particles in different levels}

There are obvious relations between the size level and 
the functions of the particles.

\subsection{The functions of the first level particles PPs}

PPs have no structural functions, which may be because they have no spatial volumes and mass, but their biological significance is important. In addition to serving as media particles in electromagnetic interactions, they can catalyze indispensible chemical and biochemical reactions in plants and lower level life bodies. Generally speaking, the functions of PPs are relatively simple although all of the life bodies can live only in dependence of the energy bought by the photons from the sun.

\subsection{The functions of the second level particles FPs}

FPs are the structural basis and module particles of all of the substance particles. They have the tiniest particle sizes with simplest or frankly unkown inner structures, of which the functions are also simplest (the fact may be that the complex functions have not been found by human). The results of their functional activities are the production of 479 FPs and the atomic nucleus of 114 chemical elements. Some FPs have important biological functions. For example, the electrons, as the bearer of electric charges as well as the media particles for the electromagnetic interactions, are in-dispossible in biochemical and biological reactions, despite they may be depended of indirectly as in the case of NPs. Another FPs such as protons and neutrons are the fundamental particles to make up atoms, which are the most important structural basis of all of the substance particles.

\subsection{The functions of the third level particles ChPs}

The functions of the ChPs manifest as complex chemical reactions. The complexity of chemical interactions and functional activities are incomparable with fundamental particles, representing a leaping, and arise from the complexity of the structures of ChPs composed of FPs. It is the functional activities of ChPs that produce myriad chemical substances, making biochemistry become an independent science field, showing the colorfulness of the chemistry world. The biological functions of ChPs are very important. Firstly, ChPs are the module particles of NPs such as proteins composed of amino acids and nucleic acids composed of nucleotides. The orders of amino acids, as ChPs, in a peptide chain determine the steric structures of a protein, which in turn determines the functions of the proteins composed of these peptides, and the series of the nucleotides determine genes, which in turn determine the properties and functions of proteins. In inorganic NPs, ChPs as module particles can determine the properties and the functions of NPs. In addition to serving as module particles of NPs, the energy production is one of the most important functions of ChPs, which lays the foundations of biological activities such as the muscle contraction, gland secretion, substance transportation through barriers and so on. The energy releasing effects also have great promise in the future nanorobots. Thirdly, ChPs may act as the function regulator of biological NPs. For example, the reactivators of receptors or enzymes improve while the blockers of receptors and inhibitors of enzymes suppress the activities of receptors and enzymes. Besides the above mentioned biological functions, ChPs have the fourth important function, that is, to act as messengers in the message transmission such as the nerve transmitter for the transmission of nervous signal through neurosynaptic gaps and the second messengers in the intracellular signal transmission, or to act as the media particles for the interactions of NPs with each other or with environments. Some smaller molecule hormones such as epinephrine and thyroxin can be transported by blood circulation to target organs, tissues, and cells to realize the remote regulation of biological or life activities. There is the fifth function of ChPs, that is, to serve as drugs to cure diseases. Some special ChPs can regulate the biological functions of proteins, genes, cells, organs, systems and even the whole life bodies.

\subsection{The functions of the fourth level particles NPs}

The fourth level particles have more complex functions with the features of biological activities of NPs. NPs are composed of ChPs but have much stronger functions as well as much more complex particle structures in comprison with their module ChPs. First, biological NPs have manufacture functions, that is, they can create ChPs through biochemical reactions such as synthesis, group-transmission, hydrolization, hydrogenation and dehydrogenation, and so on. In addition, some enzyme NPs have modification effects, in which they make other non-functional proteins become functional by adding chemical groups or change the ateric structures of other enzyme NPs. Second, NPs have transportation functions and act as drug carriers in drug delivery. There are several advantages of NPs as drug carriers including transportation effects, target effects, barrierbreaking, slow and controlled release effects, protective effects and so forth. The transportation functions of NPs are dependent of their strong adsorptive properties for drugs and their ability of escaping the macrophage of reticuloepithelial system, capable of carrying drugs from the administration sites through the long way of blood circulation to the desired sites where the curative effects develop. Third, NPs may function as messengers for remote regulation of biological or life activities. For example, insulin secreted from pancreatic islets can get through blood circulation to their effectors of the whole body to regulate the metabolism of glucose according 
to the needs of life bodies; Pituitary hormones such as parathyrotropin can get to their effector glands to regulate the secretion of various hormones according to the body needs. More complex hormone regulating systems can be built by a group of hormones. The hormones in this system have obviously different levels. Not only the hormones at higher levels can regulate the secretion of that in lower levels but also the hormones in lower levels even the final products can regulate the secretion of the hormones in the higher even the highest levels through negative feedback. Fourth, NPs composed of nucleosides function as the transmitter of heredity messages. The genes are namely the series of nucleosides in DNA molecules, which determines the trait or characters of the descendants. Fifth, NPs can function as the module particles of functional MPs. The NPs aggregated together according to strict principles to form microtubes and skeleton of cells, which in turn form the tissues of the life bodies. It may be said that the fourth level particles are such key particles that connect non-life ChPs and the life particles like a bridge. NPs are the basic units of the life bodies and the performer of the fundamental life activities. Here it may be predicted that the nanoscale is the least scale for life phenomenon to develop, and the mastery of the life will be disclosed at the size level of NPs. Those man-made non-life NPs are also getting more and more smart. For example, it has been found that some inorganic NPs can function as catalase, a very important enzyme to catalyaze the decomposition of $\mathrm{H}_{2} \mathrm{O}_{2}$ to protect cells from the damage of such toxic molecules.

\subsection{The functions of the fifth level particlesMPs}

The functional activities of the fifth level particles manifest as the functional activities of the individual life bodies. MPs composed of biological NPs in the level higher than nano-level are indeed living bodies while those inorganic MPs have various functions as machines. The functions of MPs are so strong that are in no way comparable with NPs. The life bodies may be considered as the representative MPs. There are several features in the functional activities of the life bodies. The first is the metabolism, including assimilation and dis-assimilation. Through assimilation, life bodies continually to obtain nutrients from their environment to build up themselves' bodies, and through dis-assimilation, the body structures continually disintegrate into their module particles such as amino acids, which are finally oxygenated into $\mathrm{CO}_{2}$ and water. During metabolism, the life bodies can be kept integrated. The second is heredity and self proliferation. Individual life bodies can produce decendants that are very similar with themselves through fission and regeneration. The third is their active adaptation to environment. Except plants and some low-level animals, all of the life bodies can adapt to their environment positively. The fourth is the high level nervous activities including learning, memory and thinking, which can't develop in the particles in lower levels including PPs,
ChPs and NPs. At present, the living bodies composed of the particles in the lower levels have not been found. The virus are composed of biological particles such as proteins and nucleic acids and have sizes of nanometer but they haven't the functions indispensible for their metabolism, proliferation and environment adaptation. They must complete their proliferation dependent of the enzyme systems in host cells.

\subsection{The functions of the sixth level particles CePs}

As for the functions, we still know little about the celestial bodies, but it may be said that it is the celestial bosies that bring forth the life in the earth (may be also in other planet) so we can say that giving birth of life is one of the complex functions of solar system composed of celestial bodies. In this solar system, the sun continually provide the earth with light and heat, dependent on which the lives develop and survive on earth. From this fact, one spot of the strong functions of the celestial bodies may be seen. More functions of the celestial bodies may be found with the development of sciences and technology.

\section{Summary and perspectives}

All the substance can be viewed as particles. All the substance particles can be classified according to their sizes. The substance particles in different size levels have their unqiueessential properties and functions. The present paper is the first to establish the size-function relations of substance particles from the least point particles to the largest celestial particles, which are listed in table 1.

From table 1, several particles for the size-function relations may be seen: 1) The functions of substance particles are positively related with the sizes, namely, the larger the particles, the stronger and more complex their functions are; 2) The force ranges get longer and longer with the increase of the sizes of substance particles: there are no interactions between PPs; short-range weak and strong forces govern the interactions between FPs; longrange electromagnetic forces govern the interactions of ChPs; there are electromagnetic and gravity forces to govern the interactions between NPs although the gravity is relatively less important; in the level of MPs, the roles of gravity become more important although electromagnetic forces are still the main forces to govern the interactions between MPs; between CePs, gravity becomes the main forces to govern the interactions; 3 ) all of the interactions between all of the substance particles are dependent of media particles, especially those in the lower size levels; 4) All of the particles, with exception of PPs, are composed of the particles in the levels lower than that in the levels themselves exist in; 5) the complexity of the reactions increases with the increase of the sizes of substance particles: life reactions between MPs > biological reactions between NPs $>$ chemical 
reactions between ChPs $>$ particulate reactions between FSPs. As for PPs and CePs, we still don't know whether there are any reactions between them; 6) the particles in different levels can transform from and to each other, which develop mainly between FPs and ChPs; 7) the manufacture function began from NPs and get strongest when the particle get to macroscopic size; 8) there are regulating relations between the functions of the particles in different size levels. The basic principle is that the larger particles are almost always regulated by smaller ones.

Through the systematic analysis of the functions of substrate particles, it may be seen that the nanoscale is the least scale requirement for life activities to develop. MPs manifest as the individual life bodies in the field of life sciences. The life bodies carry out functional activities of systematic metabolism, reproduction and environment adaptation of themselves. Advanced individual life bodies have advanced nervous activities. The functional activities of the CePs give birth to such miracles as life bodies. As far as the present level of natural science, human have the full understanding of MPs and most investigation into the structures, properties and functions of ChPs. The knowledge of human about PPs, NPs, and CePs are still much insufficient. The difficulties in understanding of FPs are because of their over-small, that of the CePs are of their over-large. As for NPs, it is because that we have just seen their significance in biology and life science and the studies on them have just begun and we have not spent much time on them. We say that NPs are neither too large, nor too small so that it is easier to fully understand their structures, properties, functions and the ways to manufacture them. Presently, human are endeavoring to investigate into NPs and trying to control the interactions between them. It may be said that the advent of nanotechnology has provided us an important opportunity to fully understand the life phenomenon at the nanoscale level.

Also, through systematic analysis and comparison of the substance particles we can see new challenges for scientists in the biological fields. We only outline the size-function relations of substance particles but many details remain to be clarified well which is especially important for biomedical science. What is the principle behind the interconnections and interactions between the particles in different levels? Whether those diseases with unidentified causes can be caused by the FPs? Whether those principles in different scientific fields about the particles in different levels can be lend to or borrowed from each other? How can we prepare higher size-level particles from lower size-level particles, or vice versa? Now we have known that new chemical element atoms can be obtained by the fission of nucleus resulted in by FSPs; The ChPs can be used to prepare and modify NPs, making them to obtain new properties or functions; The NPs may also be used to regulate chemical reactions and to prepare new ChPs; MPs even life bodies can be prepared from NPs; and CePs also have influences on the exist and the regeneration of MPs. The illustration of the relations between these sciences about the particles in different size-levels will lead to the final discovery of life mystery.

\section{References}

1. Y Zhang, S Guo, Z Zhang, D Cui. Substance particles. In Y Zhang, S Guo, Z Zhang, D Cui eds. Nanotoxicology. Peking Union Medical University Publishing House. Beijing. 2010.1. pp109-153.

2. S. Hawking. A Brief History of Time. Bantam Books. New York. 1988.

3. C.S. Gao, B.Q. Xie. Physics Today. High Education Press, Beijing. 2004.

4. Baughman, R. H.; Zakhidov, A. A.; de Heer, W. A., Carbon nanotubes - the route toward applications. Science 2002, 297 (5582), 787-792. doi:10.1126/science. 1060928

5. Zheludev, N. I., The Road Ahead for Metamaterials. Science 2010, 328 (5978), 582-583.doi:10.1126/science.1186756

6. Allen, T. M.; Cullis, P. R., Drug delivery systems: Entering the mainstream. Science 2004, 303 (5665), 1818-1822.doi:10.1126/scie nce. 1095833

7. P. K. Dhar. The next step in biology: A periodic table? J. Biosci. 2007, 32: 1005-1008.doi:10.1007/s12038-007-0099-8

8. Siegel, J., Inventing the nanomolecular wheel. Science 2005, 310 (5745), 63-64.doi:10.1126/science. 1118765

9. Lower, S. K.; Hochella, M. F.; Beveridge, T. J., Bacterial recognition of mineral surfaces: Nanoscale interactions between Shewanella and alpha-FeOOH. Science 2001, 292 (5520), 1360-1363.doi:10.1126/ science. 1059567

10. Shekhawat, G. S.; Dravid, V. P., Nanoscale imaging of buried structures via scanning near-field ultrasound holography. Science 2005, 310 (5745), 89-92.doi:10.1126/science.1117694

11. Dietz, H.; Douglas, S. M.; Shih, W. M., Folding DNA into Twisted and Curved Nanoscale Shapes. Science 2009, 325 (5941), 725-730. doi:10.1126/science. 1174251

12.Curran, J. M.; Chen, R.; Stokes, R.; Irvine, E.; Graham, D.; Gubbins, E.; Delaney, D.; Amro, N.; Sanedrin, R.; Jamil, H.; Hunt, J. A., Nanoscale definition of substrate materials to direct human adult stem cells towards tissue specific populations. J Mater Sci: Mater Med 21 (3), 1021-1029.doi:10.1007/s10856-009-3976-X

13.Schlichting, H. E.; Ofek, E. O.; Wenz, M.; Sari, R.; Gal-Yam, A.; Livio, M.; Nelan, E.; Zucker, S., A single sub-kilometre Kuiper belt object from a stellar occultation in archival data. Nature 2009, 462 (7275), 895-897.doi:10.1038/nature08608

14.Lee, C. H.; Lin, T. S.; Mou, C. Y., Mesoporous materials for encapsulating enzymes. Nano Today 2009, 4 (2), 165-179.doi:1 0.1016/j.nantod.2009.02.001

15.Zrazhevskiy, P.; Gao, X. H., Multifunctional quantum dots for personalized medicine. Nano Today 2009, 4 (5), 414-428.doi:10.1 016/j.nantod.2009.07.004

16. Lacerda, L.; Raffa, S.; Prato, M.; Bianco, A.; Kostarelos, K., Cellpenetrating CNTs for delivery of therapeutics. Nano Today 2007, 2 (6), 38-43.doi:10.1016/S1748-0132(07)70172-X

17.Z.Yang, Y. Zhang, Y. Yang, L. Sun, D. Han, H. Li, C. Wang. Nanomed. 2010, 6, 427.doi:10.1016/j.nano.2009.11.007

18.Pechmann, S.; Levy, E. D.; Tartaglia, G. G.; Vendruscolo, M., Physicochemical principles that regulate the competition between functional and dysfunctional association of proteins. Proc Natl Acad Sci U S A 2009, 106 (25), 10159-64.doi:10.1073/pnas.0812414106

19.G. Sgouros, J.C. Roeske, M.R. McDevitt, S. Palm, B.J. Allen, D.R Fisher, et al. J. Nucl. Med. 51(2010) 311.doi:10.2967/jnumed.108.0 58651

20. Macklis, R. M.; Kinsey, B. M.; Kassis, A. I.; Ferrara, J. L.; Atcher, R. W.; Hines, J. J.; Coleman, C. N.; Adelstein, S. J.; Burakoff, S. J., Radioimmunotherapy with alpha-particle-emitting immunoconjugates. Science 1988, 240 (4855), 1024-1026. doi:10.1126/science. 2897133

21. C. D. Claassen. Casarett \& Doull's Toxicology: The Basic Science of Poisons. 6th Edition, McGrraw-Hill, NY, 2001.

22.Zalutsky, M. R.; Vaidyanathan, G., Astatine-211-labeled radiotherapeutics: an emerging approach to targeted alpha-particle 
http://nanobe.org

radiotherapy. Curr. Pharm. Des. 2000, 6 (14), 1433-1455.doi:10.217 4/1381612003399275

23. McDevitt, M. R.; Ma, D. S.; Lai, L. T.; Simon, J.; Borchardt, P.; Frank, R. K.; Wu, K.; Pellegrini, V.; Curcio, M. J.; Miederer, M.; Bander, N. H.; Scheinberg, D. A., Tumor therapy with targeted atomic nanogenerators. Science 2001, 294 (5546), 1537-1540. doi:10.1126/science. 1064126

24. McDevitt, M. R.; Sgouros, G.; Finn, R. D.; Humm, J. L.; Jurcic, J. G.; Larson, S. M.; Scheinberg, D. A., Radioimmunotherapy with alpha-emitting nuclides. Eur. J. Nucl. Med. 1998, 25 (9), 1341-1351. doi:10.1007/s002590050306

25. Mulford, D. A.; Scheinberg, D. A.; Jurcic, J. G., The promise of targeted \{alpha\}-particle therapy. J Nucl Med 2005, 46 Suppl 1, 199S-204S

26. Jurcic, J. G., Antibody therapy for residual disease in acute myelogenous leukemia. Crit Rev Oncol Hematol 2001, 38 (1), 37 45.doi:10.1016/S1040-8428(00)00132-3

27. D. S. Wilbur. . Antibody Immunoconjugates Radiopharm. 1990, 4, 85 .

28. Hultborn, R.; Andersson, H.; Back, T.; Divgi, C.; Elgqvist, J.; Himmelman, J.; Horvath, G.; Jensen, H.; Lindegren, S.; Palm, S.; Jacobsson, L., Pharmacokinetics and dosimetry of (211)AT-MX35 $\left.\mathrm{F}(\mathrm{AB})^{\prime}\right)(2)$ in therapy of ovarian cancer - Preliminary results from an ongoing phase I study. Cancer Biotherapy and Radiopharmaceuticals 2006, 21 (4), 395-395.

29. Jurcic, J. G.; Larson, S. M.; Sgouros, G.; McDevitt, M. R.; Finn, R. D.; Divgi, C. R.; Ballangrud, A. M.; Hamacher, K. A.; Ma, D. S.; Humm, J. L.; Brechbiel, M. W.; Molinet, R.; Scheinberg, D. A., Targeted at particle immunotherapy for myeloid leukemia. Blood 2002, 100 (4), 1233-1239.

30. Wicha, M. S., Cancer stem cells and metastasis: lethal seeds. Clin. Cancer Res. 2006, 12 (19), 5606-5607.doi:10.1158/1078-0432.CCR06-1537

31. Liu, H.; Schmidt, J. J.; Bachand, G. D.; Rizk, S. S.; Looger, L. L.; Hellinga, H. W.; Montemagno, C. D., Control of a biomolecular motor-powered nanodevice with an engineered chemical switch.
Nat. Mater. 2002, 1 (3), 173-177.doi:10.1038/nmat761

32. Sussman, J. L.; Harel, M.; Frolow, F.; Oefner, C.; Goldman, A.; Toker, L.; Silman, I., Atomic structure of acetylcholinesterase from Torpedo californica: a prototypic acetylcholine-binding protein. Science 1991, 253 (5022), 872-879.doi:10.1126/science.1678899

33. Raves, M. L.; Harel, M.; Pang, Y. P.; Silman, I.; Kozikowski, A. P.; Sussman, J. L., Structure of acetylcholinesterase complexed with the nootropic alkaloid, (-)-huperzine A. Nat. Struct. Biol. 1997, 4 (1), 5763.doi:10.1038/nsb0197-57

34. Gao, L. Z.; Zhuang, J.; Nie, L.; Zhang, J. B.; Zhang, Y.; Gu, N.; Wang, T. H.; Feng, J.; Yang, D. L.; Perrett, S.; Yan, X., Intrinsic peroxidase-like activity of ferromagnetic nanoparticles. Nature Nanotechnol. 2007, 2 (9), 577-583.doi:10.1038/nnano.2007.260

35. Hess, H.; Clemmens, J.; Qin, D.; Howard, J.; Vogel, V., Lightcontrolled molecular shuttles made from motor proteins carrying cargo on engineered surfaces. Nano Letters 2001, 1 (5), 235-239. doi:10.1021/n1015521e

36. Bohm, K. J.; Stracke, R.; Muhlig, P.; Unger, E., Motor proteindriven unidirectional transport of micrometer-sized cargoes across isopolar microtubule arrays. Nanotechnology 2001, 12 (3), 238-244. doi:10.1088/0957-4484/12/3/307

37. Hiratsuka, Y.; Tada, T.; Oiwa, K.; Kanayama, T.; Uyeda, T. Q. P., Controlling the direction of kinesin-driven microtubule movements along microlithographic tracks. Biophysical Journal 2001, 81 (3), 1555-1561.doi:10.1016/S0006-3495(01)75809-2

38. Soong, R. K.; Bachand, G. D.; Neves, H. P.; Olkhovets, A. G.; Craighead, H. G.; Montemagno, C. D., Powering an inorganic nanodevice with a biomolecular motor. Science 2000, 290 (5496), 1555-1558.doi:10.1126/science.290.5496.1555

Copyright:(c) 2011 Y. Zhang, et al. This is an openaccess article distributed under the terms of the Creative Commons Attribution License, which permits unrestricted use, distribution, and reproduction in any medium, provided the original author and source are credited. 